\section{Representations of odours and odour mixtures visualized in the honeybee brain}

\section{Jasdan Joerges, Armin Küttner, C. Giovanni Galizia \& Randolf Menzel}

Institut für Neurobiologie, Freie Universität Berlin, Königin-Luise-Strasse 28-30, 14195 Berlin, Germany

Most animals depend on the identification of odours to locate food or to find mating partners. To accomplish this, the olfactory system must recognize relative concentrations of a large number of substances by analysing complex patterns of chemoreceptor activations ${ }^{1,2}$, but how these patterns are represented in the brain is not well understood. Previous studies indicated that odours evoke specific patterns of activity in olfactory sensory centres $^{3-7}$ and led to the hypothesis that single glomeruli in the olfactory bulb of mammals respond to particular receptor types ${ }^{8-10}$. We made optical recordings in vivo in the honeybee brain to investigate neuronal population responses to odorants delivered naturally to the animal. We report here that odours evoked specific spatio-temporal excitation patterns in the antennal lobe, the structural and functional analogue of the olfactory bulb". Specific ensembles of active glomeruli represent odours in a combinatorial manner. A comparison between different individuals shows remarkable similarities for a pheromone component, but not for general flower odours. Mixtures evoked patterns that were combinations of the single odorant responses. These combinations were not fully additive, however, indicating inhibitory effects on single glomeruli. Such interactions could be crucial for the formation of singular codes for complex odour blends.

Both the olfactory bulb and the antennal lobe have a glomerular structure with lateral connections between glomeruli, and process incoming signals from broadly tuned odorant receptors ${ }^{1,2,11-13}$. Recordings of glomerular output neurons in insects and vertebrates indicate distributed odour representations $\mathrm{s}^{14-17}$. Evidence accumulated from molecular biological studies suggests that receptor neurons expressing the same receptor protein converge onto single glomeruli ${ }^{8-10}$. To visualize the nature of odour-elicited activity in vivo, we made optical recordings of calcium-dependent fluorescence in the honeybee, Apis mellifera. The coding of odour mixtures and the similarity between odour representations in different individuals were of special interest.

The antennal lobe is a spherical structure with 156 glomeruli that are innervated by about 60,000 chemoreceptor axons. The glomeruli lie in one layer in the outer rind of the sphere (Fig. $1 \mathrm{~A}, \mathrm{a})^{18}$ and the preparation allowed us to image those lying on the upper half of the antennal lobe. Stimulation with odours led to spatial patterns of increased fluorescence that can be related to the neuroanatomical structure (Fig. 1A, b). The centres of activity consistently corresponded to single glomeruli. Signals reached peak values of $5 \%$ change in fluorescence over background fluorescence, allowing for single-sweep measurements. Successive stimulations with the same odour led to reproducible patterns of activity (Fig. 1A, c, d). Correlation analysis of five repeated single trials for the odour citral confirms the high consistency of the responses (average value of correlation matrix: $r, 0.85 \pm 0.02$ s.e.m., $P<0.001$; time-span between first and last trial: $1 \mathrm{~h}$ ).

A distributed odour representation implies that single glomeruli can be activated by different odours. Time courses of signals at selected glomeruli are indeed odour-specific (Fig. 1B, a). They can outlast stimulation for several seconds, presumably as a result of calcium dynamics (also, responses of antennal lobe neurons can last several seconds after odour stimulus offset $\left.{ }^{19}\right)$. Intracellular recordings from olfactory interneurons led to the suggestion that odours are not only represented as spatial activity patterns, but as patterns that evolve over time ${ }^{17,19,23}$. Although our temporal resolution is too low to reveal fast dynamic effects, we could observe slower spatial dynamics that were odour-specific. Changes of spatial activity distribution over time were also reported in the salamander olfactory bulb? An example for the odour hexanol, where different glomeruli are activated in temporal succession $(\Delta t=500 \mathrm{~ms})$ is given in Fig. 1B, b.

The patterns were specific for every odour tested, with distributed but overlapping activities (Fig. 2), confirming that single glomeruli may participate in activity maps of different odours. The natural plant extract carnation, which is a complex blend of many substances, did not lead to more extensive activity than the other five pure substances tested here.

To determine whether the odour-specific glomerular activity patterns are conserved between individuals, we compared the signals of different bees (Fig. 3). Citral led to strong medial activation and to similar overall activation patterns $(n=8)$ (Fig. 3a). The minor differences could depend on slight variations in view and preparation. The representation for citral, therefore, seems to be conserved between individuals. For the tested flower odorants,
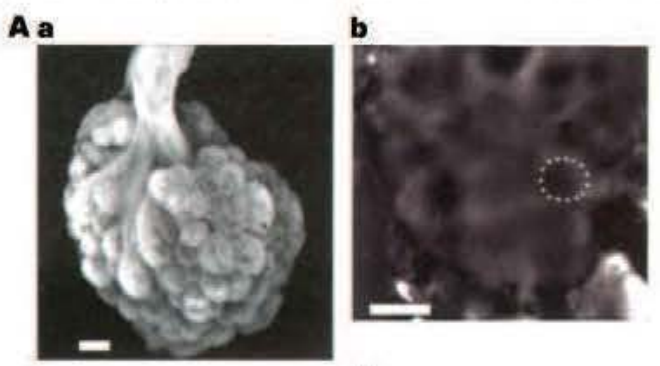

c

d
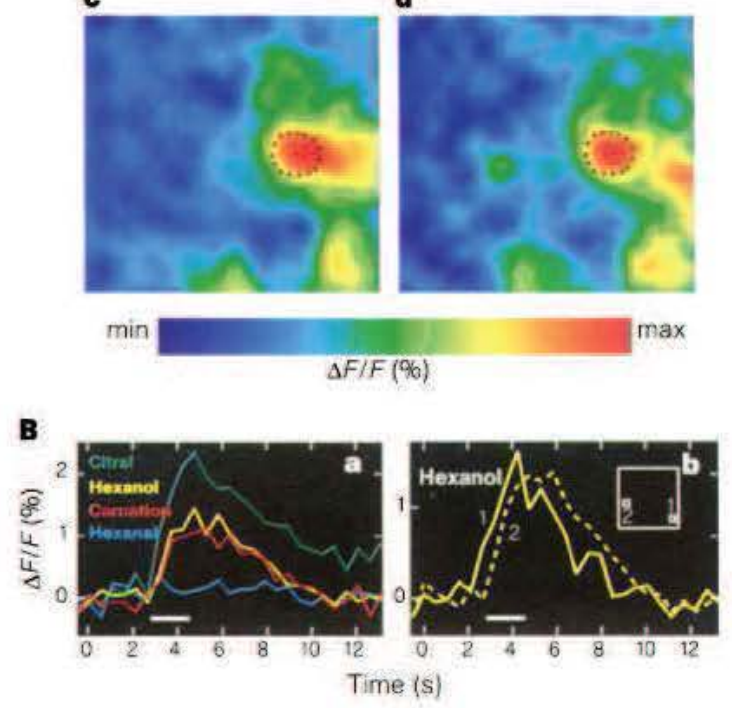

Figure $1 \mathrm{~A}$. Optical imaging during stimulation with odours yields spatial activity patterns that are odour specitic and can be related to the glomerular morphology of the honeyoee antennal lobe (AL). a, Reconstruction of an AL. b. Fluorescence picture of the imaged area from an AL stained with the dye RH795. A single glomerulus is marked with a dotted circle. Scale bars, $50 \mu \mathrm{m}$. c, Odour-evoked activity in the AL shown in b during a single 2-s stimulation with cirral (1sttrial). The outline of the glomerulus marked in $\mathbf{b}$ is superimposed (dotted line). Signai range

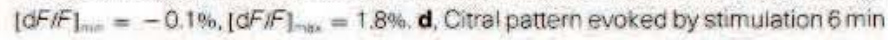
later (3rd trial with citral, 8 th trial in total, $[\mathrm{d} F / F]_{\min }=0.1 \%$, (dFIF $]_{\max }=1.5 \%$ ). Correlation between patterns in $\mathbf{c}$ and $\mathbf{d} . r=0.92(P<0.001)$. B. Time courses of caicium signals at selected glomeruli (different experiment) a, Odours elicit specific signals upon stimulation (bar). b. Hexanol leads to successive activation of different regions (see inset) 
the variability between individuals is higher, as shown for hexanol (Fig. 3b) ( $n=5$ ). We computed the correlation coefficients for all possible pairs of patterns shown (see Methods). For citral, the average correlation coefficient is $r=0.50$, whereas for hexanol, $r=0.35$. The higher correlation coefficient for citral shows that citral induces more constant responses than hexanol. This difference could be related to the fact that citral (besides being a component of some flower fragrances) is an important pheromone component for honeybees. In male moths, specialized macroglomeruli are exclusively concerned with processing sex pheromone signals ${ }^{21}$. Worker honeybees are female and, although pheromones are important for their communication, do not possess a macroglomerular complex. The patterns evoked by citral (and other pheromones, for example isoamyl acetate; see Fig. 2) overlap considerably with those of other tested odours, indicating that certain pheromones are processed by the same neural circuits as general odours.

The olfactory system must provide a concentration-invariant code for odour identity. Bees, for example, recognize odours over a wide range of concentration ${ }^{22}$. We investigated the effect of odour intensity by comparing signals evoked at different concentrations. In all cases, patterns evoked by low concentrations were topologically nearly identical to those evoked by high concentrations, but with reduced signal amplitude. For citral, the correlation between high and low concentration responses was $r=0.90, P<0.001$; for hexanol it was $r=0.83, P<0.001$. The correlation between citral and hexanol responses at high concentrations was small $(r=0.29$, $P<0.01$ ), and at low concentrations there was no significant correlation between them. Using higher amounts of odour substance $(>10 \mu l)$ led to higher signal amplitudes, showing that our

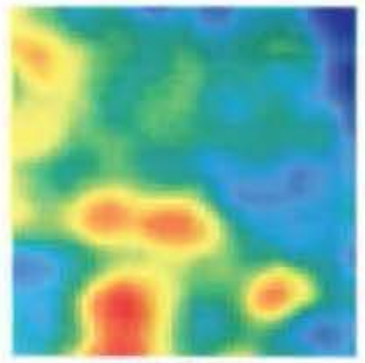

Geraniol

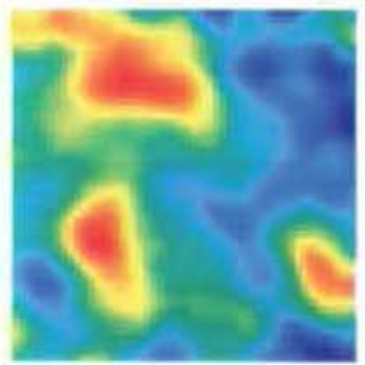

Hexanal

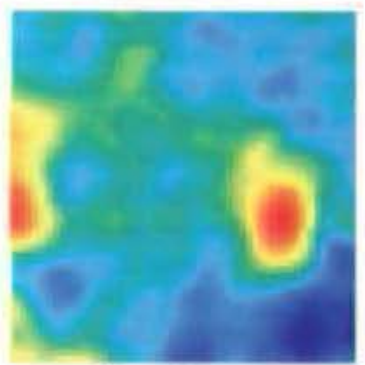

Carnation

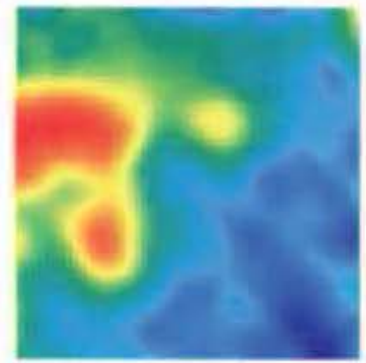

Isoamylacetate

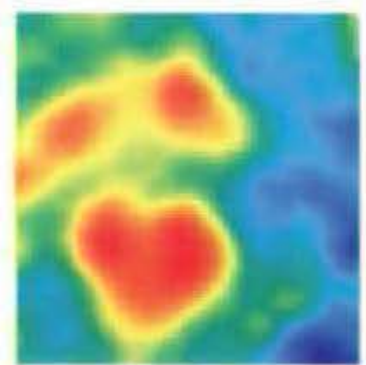

Hexanol

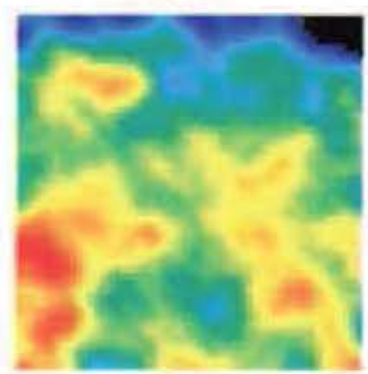

Citral standard concentration does not lead to saturation. Our findings show that concentration-invariant patterns of activity already occur at the level of the antennal lobe glomeruli, as indicated by results from the salamander olfactory bulb?

Most natural odours are complex blends of volatile compounds, so we studied the patterns evoked by mixtures of odorants. Figure $4 \mathrm{a}$ shows the results from stimulation with citral, hexanol and their binary mixture. The mixture pattern included components of both single odours. The reaction profiles shown in Fig. $4 \mathrm{~b}$, however, indicate inhibitory interactions at several glomeruli for the mixture response. Mixtures of more than two components led to stronger inhibitory effects. The results in Fig. $4 c$ show that for binary mixtures the inhibitory effects are moderate. For the ternary mixture the inhibitory effects increase, leading to a pattern that differs more from the arithmetic sum of its components than for binary mixtures.

Membrane-permeant and dextran-coupled calcium dyes were used previously in vertebrate preparations ${ }^{23,2431}$. We have succeeded for the first time in registering spatio-temporal calcium signals from a central brain structure in vivo during natural stimulation. We show that odours are represented in the antennal lobe by specific maps of active glomeruli ${ }^{1.3 .5}$. The acetoxymethyl-ester-coupled dye is likely to be taken up by receptor cells, local interneurons, projection neurons and glial cells. It is important to have some information on the origin of the signals measured. The dense set of afferents from the antenna (Fig. $1 \mathrm{~A}$, a) constitutes the largest group of neurons innervating the antennal lobe and these axons exclusively arborize in the rind of glomeruli, whereas other neurons branch in all parts of the glomeruli. This indicates that the afferents

Figure 2 Spatial activity pattems are specific for each tested odout. Six tested odours evoked distributed but overlapping glomerular activity. Neighbouring giomeruli can react quite differently to odours (see central region for hexanol and hexanal, where two glomeruli are active for hexanol, but only one is active for hexanal). Signal ranges (4th trial for each odour) geraniol, $1 \mathrm{~d} F \mathrm{FF}$. $=-0.19 \%$

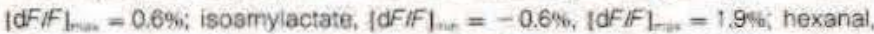

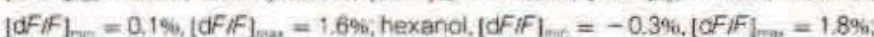

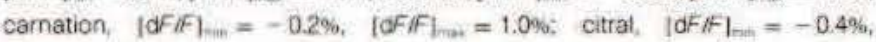
$\left.|\mathrm{d} F| F\right|_{\min }=0.7 \%$.

a

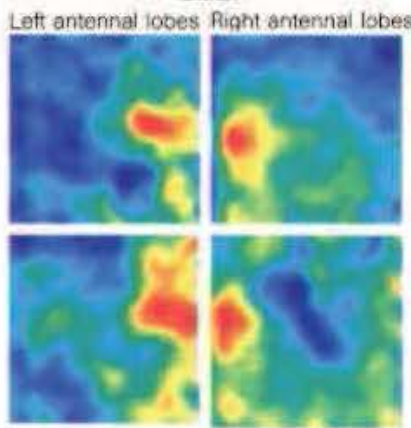

b Left antennal tobes Right antennal lobes

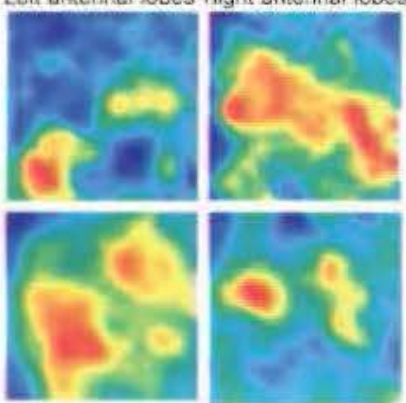

Figure 3 Patterns are more constant in different animals for the pheromone component citral than for the flower odorant hexanol, a, Citral patterns from 4 individual honeybees (2 left and 2 right ALs) all showing activity on the central medial side of the AL. Parterns are symmetrical across the longitudinal body axis.

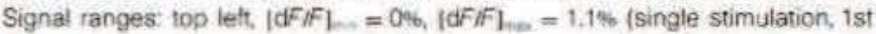

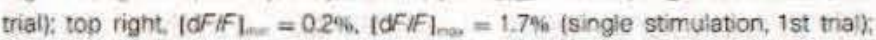
bottom lett, $\mid \mathrm{d} F / F]_{\text {.at }}=0 \%,[\mathrm{~d} F / F]_{\mathrm{w}}=0.3 \%$ (trials $1-8$ averaged) bottom right.

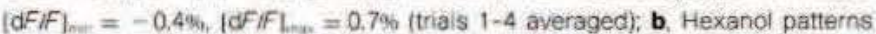
from the same 4 animals (same views of the $A L$ ) are more vanable across individuals (see text). Signal ranges (number of averaged trials as for corresponding citral patterns): top left, $[\mathrm{d} F / F]_{n n}=-0.3 \%,[\mathrm{~d} F / F]_{\text {nas }}=0.9 \%$; top right.

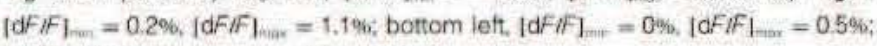
bottom right, $[\mathrm{d} F / F]_{\min }=-0.2 \%,\left[\mathrm{~d} F /\left.F\right|_{\max }=1 \%\right.$ 
a

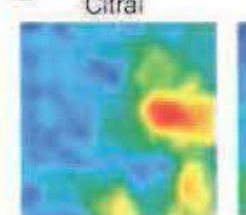

Mix citral + Mix citral
hexano

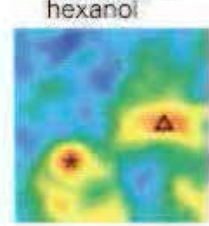

$\Delta$

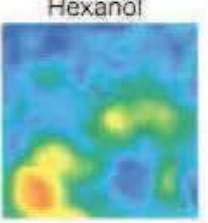

Caiculated sum

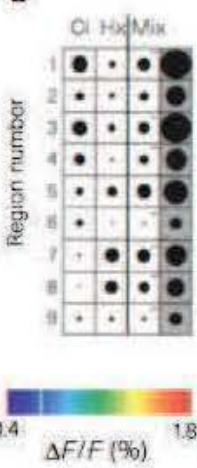

c

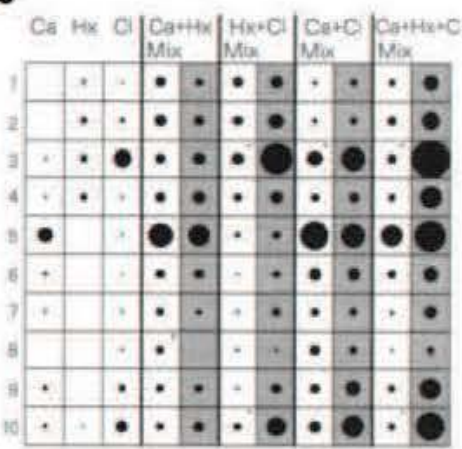

Figure 4 Mixtures of odorants elicited patterns that include components of the single odorant patterns. a, Patterns evoked by citral, hexanol and by their binary mixture. For comparison, the arthmetic sum of the two single response panterns is shown. The glomerulus marked with a triangle in the mixture pattern was less active than during stimulation with citral alone. The star marks a glomerulus that was more active than expected by the arithmetic sum (1st tria). b. Response profiles of 9 different glomeruli (same experiment as in a). Size of circles gives maximal signal in response to odours (smallest circle corresponds to

contribute a major part to the signal. Mixture interactions between odorants are likely to occur at several stages in the olfactory pathway starting with the receptor cells ${ }^{25}$. GABA-immunoreactive interneurons of the antennal lobe may also synapse back onto receptor cell terminals, as has been shown in the cockroach Periplaneta american $a^{26}$. Consequently, the observed nonlinear mixture effects could be the result of interactions at the receptor level, or of network interactions in the antennal lobe ${ }^{15,27}$, or both. In all cases they could be important for the formation of distinct olfactory codes for given odour blends. Even though olfactory receptor cells can have highly nonlinear reaction properties ${ }^{25.28}$, the patterns were almost independent of stimulus concentration? We conclude that the antennal lobe is capable of forming stable representations from complex sensory responses ${ }^{2}$. Results from molecular biological studies in vertebrates have shown consistent and mirror symmetric projection patterns of olfactory receptors to the olfactory bulb in different individuals ${ }^{8,910}$. The functional activity patterns shown here demonstrate invariance and mirror symmetry for a behaviourally important pheromone. For non-pheromones, the inter-individual differences are higher. A possible hypothesis is that their representations may be modified on the basis of individual experience. Neural plasticity in olfactory neuropiles can lead to changes in behaviour, both in insects and vertebrates ${ }^{30}$. Future experiments, combining functional imaging of the intact brain with olfactory learning, will provide a chance to investigate the formation of olfactory memories in vivo.

\section{Methods}

Animals. Adult worker honeybees (departing from the hive) were caught in the morning, fixed in metal tubes and fed with sucrose solution. Animals were experimentally naive, but may have learned odours in the field before being captured.

Preparation, staining and imaging. Calcium-dependent fluorescence was used as an indicator of neuronal activity. Experiments were performed with adult worker bees (in some cases immobilized heads only). The brain was incubated with the calcium indicators calcium-green-1-acetoxymethyl (AM) or calcium-green-2-AM (Molecular Probes, Oregon) for $1 \mathrm{~h}$, resulting in uniform labelling of the antenal lobe (AL). Images of the AL were taken from the intact brain perfused with bee saline through a window cut in the head capsule covered with a cover glass. About 30 of 156 glomeruli in the AL were imaged simultaneously. Clear signals were registered in 27 bees for up to $4 \mathrm{~h}$. Signals are expressed as a change in fluorescence over background fluorescence $(\mathrm{d} F / F)$. Series of $30-50$ frames $\{2-3$ frames per s) starting $4-6 \mathrm{~s}$ before stimulation were recorded with a CCD camera (Photometrics CH250A).
$\mathrm{dFIF}=0.3 \% ;$ largest circle, $\mathrm{dF} / \mathrm{F}=2.1 \%$; signals $<0.3 \%$ excluced). Activities of citral $(\mathrm{Ci})$ and hexanol $(\mathrm{Hx})$ are combined in the mixture response (Mix). Minus signs mark sites where mixture activity is less than the largest single component activity. The erithmetic sum of $\mathrm{Cl}$ and $\mathrm{Hx}$ is shown with grey background. $\mathbf{c}$, Response profiles from another experiment with carnation (Ca), hexanol and citral. The 3 possible binary mixtures and the ternary mixture are shown as well ( $d F_{i F_{\min }}=0.2 \%$, CFiF $=1,4 \%$; trials $1-4$ averaged).

Exposure time per frame was $200 \mathrm{~ms}$. The confocal reconstruction shown in Fig. $1 \mathrm{~A}$, a was prepared by injecting neurobiotin into the antennal nerve and staining with streptavidin/CY-3.

Odour stimulation. Odours were delivered to the freely moving antennae using a custom-made olfactometer by switching from a constant stream of air to an odorant containing one in order to eliminate mechanical stimulation associated with stimulus onset. Stimulus duration was $2 \mathrm{~s}$. Concentrations were determined by the amount of odorant substance placed on filter paper inserted in the olfactometer. Pure concentration corresponds to $5 \mu \mathrm{l}$ substance, 1:100 corresponds to $5 \mu \mathrm{l}$ substance diluted 1:100 in mineral oil. Mixtures were produced in the olfactometer by mixing two odorant-containing air streams (the same results were obtained by using a mixture prepared in advance and placed inside one odour container). To ensure that we used physiological odour concentrations, we trained bees to odours with the same olfactometer (in separate experiments). Animals responded clearly and selectively only to the trained odour (data not shown).

Figures and statistics. Images were spatially low-pass filtered with a $7 \times 7$ pixel pyramidal filter. No temporal filters were applied. Data were corrected for dye bleaching by subtracting one sweep without stimulation (background trial) from one with stimulation. For all figures, 3-6 frames during the 2-5 stimulation interval were averaged to give a single frame. These were false-colourcoded to show the relative increase of calcium-dependent fluorescence showing the odour-evoked response. For correlation analysis, the linear Pearson correlation coefficient between two frames was calculated on spatially binned data $(7 \times 7$ pixel). Significances are given for two-sided tests. (Applying Spearman's rank correlation led to equivalent results.) Where measurements of left and right side of the brain were compared (Fig. 3), the mirror image of the left-side patterns and the unmodified right-side patterns were used to calculate correlations to account for the mirror symmetry of left and right. The arithmetic sum pattern shown in Fig. 4 a was rescaled to the same range as the measured mixture pattern to allow direct comparison.

1. Shepherd, G. M. in Offaction. A Model Sysum for Compuational Neurostience (eds Davis, I. \& Fichenbaum, H.) 3-41 (MIT Press, Cambridge, MA, 1991).

2. Iaurem, G. Odor Images and Tanes. Nearon 16, 473-476 (1996).

3. Stewart, W. B.. Kauer. I. S. \& Shepherd. G, M. Functional organization of tat olfactory bulb analyzed by the 2-deoxyglucose methad. I Comp. Neurol, 185, 715-734 (1979).

4. Kauer, I. S. Real-time imaging of evoked activity in local circuits of the salamander olfectory bulb. Nature 331, $166-168$ (1988):

5. Rodrigues, $\mathrm{V}$. spatial coding of ollactory information in the antennal lobe of Drosuphila melanegasten. Brain Res. 453, 299-397 (1988)

5. Lieke, E. Optical tecording of neuronal activity in the insect central nervous system: odorant coding by the antennal fobes of honeybees. Fur. I. Neurosit. 5, 49-55 (1993).

7. Cinclh, A. R., Hamilton, K. A. \& Kauer, 1. S. Salamander olfactory bulb neuronal activity observed to video rate, voltage-sensitive dye imaging. 111 . Spatial and temporal properties of responses evoked by odorant stimulation. 1. Neurophysiol. 73, 2053-2071 (1995). 
8. Vassar, R. et al. Topographic organization of sensory projections in the olfactory bulb. Cell 79, 981 991 (1994).

9. Ressler, K. J. Sullivan, K. J, \& Buck, L. B. Information coding in the olfactory system: evidence for a stereotyped and highly organized epitope map in the olfactory bulb. Cell 79, 1245-1255 (1994).

10. Mombaerts, P. et al. Visualizing an olfactory sensory map. Cell 87, 675-686 (1996)

II. Boeckh, J., Distler, P., Ernst, K. D., Hösl, M. \& Malun, D. in Chemosensory Information Processing (ed. Schild, D.) 201-227 (Springer, Berlin, 1990).

12. Firestein, S., Picco, C. \& Menini, A. The relation between stimulus and response in olfactory receptor cells of the tiger salamander, J. Physiol. 468, 1-10 (1993).

13. Schild, D. Signal integration in the olfactory system. Trends Nesurosci. 17, 366-367 (1994).

14. Christensen, T. A., Waldrop, B. R., Harrow, I. D. \& Hildebrand, I. G. Local interneurons and information processing in the olfactory glomeruli of the moth Manduca sexta. J. Comp. Physiol. A 173, 385-399 (1993).

15. Sun, X., Fonta, C. \& Masson, C. Odour quality processing by bee antennal lobe interneurones. Chem. Senses 18, 355-377 (1993).

16. Mori, K. \& Yosthihara, Y. Molecular recognition and olfactory processing in the mammalian olfactory system. Progr. Neurobiol, 45, 585-619 (1995).

17. Wehr, M. \& Laurent, G, Odour encoding by temporal sequences of firing in oscillating neural assemblies. Nature 384, 162-166 (1996).

18. Flanagan, D. \& Mercer, A. R. An atlas and 3-D reconstruction of the antennal lobes in the worker honey bee, Apis mellifera. Int. I. Insect Morphol. Embryol. 18, 145-159 (1989).

19. Christensen, T. A.. Hildebrand, I. G., Tumlinson, J. H. \& Doolittle, R. E. Sex pheromone blend of Manduca sexta. responses of central olfactory interneurons to antennal stimulation in male moths. Arch. Insect Biachem. Physiol. 10, 281-291 (1989).

20. Tank, D. W., Gelperin, A. \& Kleinfeld, D. Odors, oscillations, and waves: does it all compute? Science $265,1819-1820\{1994)$

21. Hanson, B. S., Ljungberg, H., Hallberg. E. \& Löfstedt, C. Functional specialization of olfactory glomeruli in a moth. Science 256, 1313-1315 (1992).

22. Pelz, C., Gerber, B. \& Menzel, R. Odorant intensity as a determinant for olfactory conditioning in honeybees: roles in discrimination, overshadowing and memory consolidation. I. Exp. Biol. 200, 837847 (1997).

23. Yuste, R. \& Katz, L. C. Control of postsynaptic $\mathrm{Ca}^{-1}$ influx in developing neocortex by excitatory and inhibitory neurotransmitters. Neuron 6, 333-344 (1991).

24. O'Donovan, M. J., Ho, S., Sholomenko, G. \& Yee, W. Realtime imaging of neurons retrogradely and anterogradely labelled with calcium-sensitive dyes. J. Neurosci. Meth. 46, 91-106 (1993).

25. Akers, R. P. \& Getz, W. M. Response of olfactory receptor neurons in honey bees to odorants and thei binary mixtures. 1. Comp. Physiol. A 173, 169-185 (1993).

26. Distler, P. GABA-immunohistochemistry as a label for identifying types of local interneurons and their synaptic contacts in the antennal lobes of the american cockroach. Histochemistry 93, 617-626 (1990).

27. Hansson, B. S., Anton, S. \& Christensen, T. A. Structure and function of antennal lobe neurons in the male turnip moth, Agrotis segetum. J. Comp. Physiol, A 175, 547-562 (1994).

28. Ache, B. W. Towards a common strategy for transducing olfactory information. Semin. Cell Biol. 5 55-63 (1994).

29. Hammer, M. \& Menzel, R. Learning and memory in the honeybee. I. Neurosci. 15, 1617-1630 (1995)

30. Kendrick, K. M., Levy, F. \& Keverne, E. B. Changes in the sensory processing of olfactory signals induced by birth in shcep. Science $256,833-836$ (1992)

31. Friedrich, R. W. \& Karsching, S. I. Neuron $833-836$ (in the press).

Acknowledgements. We thank M. Harnmer, M. Giurfa, R. Yuste and A. Borst for helpful comments on the manuscript and B. Corrette for help with the English. We are grateful to T. Faber for experimental support and to S. Mcllwrath for providing the preparation of the antennal lobe shown in Fig, IA,a.

Correspondence and requests for materials should be addressed to J.J. (e-mail: joerges@neuro.biologie. fu-berlin.de). 